\title{
La disertación en la enseñanza de la filosofia: definición, procedimientos y escritura
}

\author{
Miguel Ángel Gómez Mendoza* \\ Universidad Tecnológica de Pereira
}

A partir de la definición y contextualización de la disertación como un género y procedimiento didáctico en la enseñanza de la filosofía, se considera a esta como un texto y un discurso que se fija a través de la escritura, y nos conduce a reflexionar sobre el sentido y la importancia de este trabajo de escritura con relación a la exigencia filosófica. La reflexión que se propone no es tanto la de un ejercicio formal, sino el ejercicio de una experiencia de estar en el mundo, de sí mismo y de pensamiento. No es suficiente la explicación técnica de la disertación a los estudiantes, ella también debe ser explicada filosóficamente, no solo en su sentido escolar, sino también como experiencia personal del pensamiento y escritura a partir de las condiciones de estar en el mundo.

Palabras clave: disertación, enseñanza de la filosofía, escritura, pensamiento, estudiante.

\section{THE DISSERTATION IN THE TEACHING OF PHILOSOPHY: DEFINITION, PROCESSES AND WRITING}

The dissertation based on the definition and contexts as a genre and procedural learning in the teaching of philosophy, sees this as a text and a discourse that is fixed through writing, and leads us to reflect on the meaning and the importance of this task of writing concerning the philosophical requirement. The reflection that is proposed is not so much a formal exercise, but the exercise of an experience of being in the world, of oneself and thought. It is not enough explanation of the technical presentation to the students; she must also be explained philosophically, not only in its schools, but also as a personal experience of thinking and writing from a position of being in the world.

Key words: dissertation, teaching of philosophy, writing, thinking, student.

* Doctor en Educación, Université de Paris III (Sorbonne-Nouvelle), Francia, 2001. Profesor Titular de la Universidad Tecnológica de Pereira y actual Director del Departamento de Pedagogía de la Facultad de Ciencias de la Educación de la UTP. 
partir de la definición y contextualización de la disertación como un género $\triangle$ y procedimiento didáctico en la enseñanza de la filosofía, se considera a 1 esta como un texto y un discurso que se fija a través de la escritura, y nos conduce a reflexionar sobre el sentido y la importancia de este trabajo de escritura con relación a la exigencia filosófica. La reflexión que se propone no es tanto la de un ejercicio formal, sino el ejercicio de una experiencia de estar en el mundo, de sí mismo y de pensamiento. No es suficiente la explicación técnica de la disertación a los estudiantes, ella también debe ser explicada filosóficamente, no solo en su sentido escolar, sino también como experiencia personal del pensamiento y escritura a partir de las condiciones de estar en el mundo.

\section{1. ¿Qué es una disertación filosófica?}

En el contexto educativo francés ${ }^{1}$ la disertación constituye una de las posibilidades propuestas a los candidatos al examen de estado de bachillerato o liceo; ella está en el centro de las preocupaciones tanto de los profesores como de los alumnos. Sigue siendo, en un gran número de disciplinas, una prueba mayor de los exámenes y concursos universitarios. En la tradición de la enseñanza francesa, la longevidad secular de este ejercicio y las dificultades que encuentran en él los alumnos podrían sugerir la idea de una inadecuación de esta prueba a los alumnos actuales y conducir a afirmar la necesidad de su relativización e, incluso, de su superación².

Según Michel Tozzi ${ }^{3}$ la disertación es la vía inevitable del aprendizaje de la filosofía en el último grado del liceo francés. Se pregunta, si acaso ¿es posible utilizar otros géneros practicados por los filósofos mismos como el aforismo, la carta, el dialogo? Agrega que el lugar de lo escrito es predominante en la enseñanza de la filosofía: el texto es la huella por la cual nos es transmitida la gran tradición del pensamiento; la disertación es el ejercicio mediante el cual el alumno aprende a pensar por sí mismo; es el escrito que constituye la prueba de examen, que se prepara desde el mismo inicio del último año de liceo ("terminale") ${ }^{4}$. Las exigencias de cohesión y de coherencia

1 Raffin, F (Coord) La dissertation philosophique. La didactique á l'oeuvre. INRP, CNDP, Hachette, Paris. 1994.

2 En su artículo «Faut-il sauver la dissertation ?» Jean Jacques Ginchard, resume los debates internos relativos a la disertación de los profesores de filosofía en los liceos franceses, llevados a cabo en noviembre de 2000, en tres "discrepancias" corporativas: $<<(1)$ partidarios de la disertación como "acto mismo del filosofar" contra aquellos que la definen más ampliamente y que la desencajan, esto es, se interesan en otras formas pedagógicas, filosóficas o no, pero emparentadas; (2) teóricos de la disertación como creación mediadora, "despliegue del concepto" contra aquellos de una construcción ya predefinida en la enseñanza, de tal manera que la argumentación puede muy bien ser un montaje inteligente de tesis bien comprendidas; (3) finalmente, profundamente, profesores de filosofía, "filósofos", que asumen la soledad de su materia y de su oficio para que luego de la disertación, concluir que hay una vida después de la filosofía (y adelante!)! > En: L'agora. Revue Internationale de didactique de la philosophie. $N^{\circ} 11$. (Traducción libre mía).

3 Tozzi, Michel "Diversifier l'écriture philosophique" en: L'agora. Revue Internationale de didactique de la philosophie. $N^{\circ}$ 7. 2000. (Recuperado el 9 de agosto de 2007 en: htttp://www.crdp.montpellier.fr/ ressources/agora/ag07_032.htm).

4 Michel Onfray considera dos «suplicios» posibles en las pruebas de filosofía para el bachillerato: el 
propias de los procesos de redacción se expresan fuertemente en las necesidades discursivas del texto filosófico: preocupación por la paráfrasis de la definición de palabras, y distinciones semánticas de las nociones por la conceptualización, relaciones estrechas entre conceptos y ajuste de las preguntas en la problematización, encadenamiento ceñido de las frases y de los parágrafos en la preocupación lógica de la argumentación. La escritura es de esta manera indispensable para la precisión y el rigor de un pensamiento que trabaja sobre y por una lengua natural.

Ahora bien, según el autor francés ${ }^{5}$, cabe la pregunta, ¿si tiene en cuenta este interés de la escritura por un pensamiento filosófico, la disertación es ella, entonces, el modo privilegiado del aprendizaje del filosofar? Esta es la tesis institucional escolar: "la disertación es la forma más personal y la más elaborada del trabajo del alumno de filosofía" . Ella es "tarea de la razón, figura de la enseñanza filosófica"7; la única "forma de evaluación escrita coherente con una enseñanza filosófica" Tozzi, la oposición declarada frente a la propuesta de Alain Renaut (presidente del "Grupo Técnico Interdisciplinar", GTD, de filosofía, grupo encargado, por designación del ministerio de educación, de elaborar los proyectos de programas para los liceos de enseñanza general y tecnológica de Francia) de introducir, junto a la disertación, una confrontación de dos textos de filosofía sobre una misma pregunta9.

comentario de texto y la disertación. Sobre esta última anota: "Segundo suplicio posible: la disertación. Condición previa para minar vuestra moral. Habéis optado por el ejercicio que es la gloria de la enseñanza francesa, pero también la ocasión más frecuente de desesperar: gloria, porque durante mucho tiempo este ejercicio ha servido para seleccionar las élites y los dirigentes, la disertación ha sido el instrumento de selección por excelencia. Sin embargo, se ha convertido en la ocasión de desesperar al cuerpo de profesores; hay que confirmar que este ejercicio está mal adaptado a las condiciones del mundo y de la enseñanza de hoy en día, de manera que pocos alumnos llegan a destacar en él. Y nosotros sufrimos al corregiros e infligiros, casi siempre, malas notas o muy mediocres. Casi todas las notas académicas y nacionales se encuentran entre 6 y 11 sobre 20. Por debajo del 6 se baja a la mínima nota del profesor-que puede llegar hasta 1 ó 2 ....Por encima de 11 se reparten las buenas notas, un pequeño paquete hasta la nota más alta - nunca 20 (al contrario que en matemáticas...), de hecho entre 11 y 11 ó 16." (Antimanual de filosofía. Lecciones socráticas y alternativas. Editorial EDAF-Ensayo. Madrid, 2005. Prólogo de José Antonio Molina. Traducción del francés de Irache Gamuza Fernández. Original: Antimanuel de philosophie. Editions Breal. Paris. 2005.

5 Ibíd., p. 1

6 Circulaire fondatrice de 1925. Citado por Tozzi.

7 Dreyfus, Inspection Générale, 1995. Citado por Tozzi.

8 Bulletin d'adhésion de l'Association des professeurs de Philosophie, 1998. Citado por Tozzi.

9 Philippe Meirieu, refiriéndose a la disertación aprecia que: "Si nos referimos a la evaluación, la disertación, el texto de 750 palabras, el peligro, parece, ser el de encerrar en un ejercicio sobre el cual se podría, de una manera formal, esperar obtener un $100 \%$ de logro sobre los criterios formales, olvidando que no se trata de un formalismo y que lo que es importante en realidad es precisamente, lo que yo no puedo evaluar. El dominio de las técnicas no es suficiente. Nuestro papel de educadores es por lo tanto actuar sobre estas condiciones (...) Quisiera en tanto pedagogo, destacar otro problema: la reducción del filosofar a un solo tipo de técnica. ¿La historia de la filosofía no muestra que filosofar sea exclusivamente la redacción de disertaciones? Me parece que la fijación excesiva sobre un cierto tipo de técnica (en este caso, la disertación) conduce a una banal integración, por los alumnos, del formalismo escolar en detrimento de una real inscripción en la actividad fundadora. Hay alumnos capaces de hacer excelentes disertaciones de filosofía, perfectamente conformes a todas las tipologías y a todos los criterios que se puedan establecer, y donde no hay un ápice de filosofía, es decir que no testimonian en nada una actividad personal de pensamiento libre y de pensamiento crítico" en: $<<$ Dialogue avec des professeurs de philosophie sur "l'approche par competences en éducation.»>> (Recuperado el 10 de agosto de 2007 en: http:// www.meirieu.com) (Traducción libre mía). 
Para Russ ${ }^{10}$, la disertación filosófica tiene su especificidad, es un "ejercicio espiritual": «La disertación filosófica, irreductible a una demostración matemática, se encuentra próxima en ciertos aspectos, a un ejercicio espiritual: designa una actividad del pensamiento y un itinerario "reflexivo"; en la disertación exitosa, el estudiante procede a un diálogo consigo mismo y, al término de su decurso, se muestra capaz de cambiar de punto de vista y de convicción. Ha realizado efectivamente un "ejercicio espiritual". El hecho mismo de hablar de "ejercicio filosófico" no es, sin duda alguna, un accidente. Realizar un trabajo filosófico, disertación o comentario es ejercitar el pensamiento, dominarlo y al mismo tiempo cambiarse uno mismo. Lo que cuenta es la realidad misma del ejercicio, la capacidad de quien reflexiona para instalarse en el corazón de los problemas y convivir con ellos, hasta que se haga la luz».

Se trata entonces con la disertación de contribuir a formar el pensamiento antes que de informarse y acceder a soluciones particulares. Igualmente, para la autora ${ }^{11}$ la disertación filosófica es una "demostración argumentada" emparentada con el "ejercicio espiritual", que se define por su especificidad y que designa: «una demostración progresiva, rigurosa y coherente, que en absoluto descuida la trama de la argumentación. Esta demostración opera a partir de un problema definido con anterioridad que surge del examen de un cuestionamiento orientado y organizado, acerca de un enunciado. Este cuestionamiento organizado se inserta y se integra en un proceso global: la problemática. Se trata, por tanto, de estructurar bien un conjunto de cuestiones subyacentes al enunciado, de vincularlas de forma lógica, de circunscribir la cuestión fundamental y decisiva inherente al tema y, mediante una "argumentacióndemostración" coherente, responder, progresivamente, a los elementos inscritos en la problemática, de forma que se llegue a la determinación y a la "resolución" del problema, resolución que excluye toda rigidez, toda solución dogmática. Concluir es aportar una respuesta, tomar una posición, realizar un cierto balance sin nunca petrificar la vida ni dogmatizar (lo que sería contrario con la interrogación filosófica). La disertación filosófica puede, pues, definirse como un itinerario móvil y dinámico, que llega a una conclusión clara, a través de una problemática y una discusión organizada. » (Cursivas mías)

\section{2. ¿Qué no es una disertación filosófica?}

Para Russ ${ }^{12}$, entonces la disertación filosófica representa un ejercicio privilegiado de enseñanza. ¿Cómo definirla? Se puede entonces intentar definirla por lo que no es la disertación y así responder, complementar y especificar un poco más la definición que se ha expuesto hasta ahora. ${ }^{13}$

(1) no es un ejercicio de erudición. Muchos estudiantes no captan la especificidad de este proceder filosófico. En efecto, en filosofía no se trata de proceder por pura erudición,

10 Russ, J., Los métodos en filosofía. Editorial Síntesis, Madrid, 2001, P. 93. Traducción y adaptación de Francisco Javier Vidarte, del original francés: Les méthodes en Philosophie, Armand Collin Éditeur, Paris, 1997.

11 Ibíd., p. 99.

12 Russ, J., Los métodos en filosofía. Editorial Síntesis, Madrid, 2001, P. 93. Traducción y adaptación de Francisco Javier Vidarte, del original francés: Les méthodes en Philosophie, Armand Collin Éditeur, Paris, 1997.

13 Ibíd., Pp. 93-99. 
sino de saber reflexionar y plantear bien los problemas;

(2) no es un desfile de conocimientos, porque se corre el riesgo de convertir el razonar en una recitar y relacionar doctrinas, autores y teorías diversas. Se trata con la disertación, ante todo, de integrar los conocimientos y los saberes en el seno de una reflexión libre y personal;

(3) no es ejercicio literario, porque es igualmente irreductible a un género literario. Quizás la diferencia está en que en la enseñanza de la filosofía a través de la disertación la actividad del pensamiento procede mediante el "trabajo del concepto"; la disertación filosófica se mueve, aunque los ejemplos y las ilustraciones concretas estén autorizados e incluso deseables, en el campo puro de la abstracción. Se trata, en primer lugar, de un proceso riguroso de clarificación de conceptos para llegar a una conclusión, mientras que el campo literario pertenece más al ámbito de lo concreto y lo imaginario;

(4) no es un ensayo ${ }^{14}$. Este se define como una obra en prosa, de factura libre, acerca de un tema que no llega a agotar, constituido muy frecuentemente por una serie de reflexiones o de meditaciones en torno a un tema. El ensayo, puede entenderse como el conjunto de una mezcla erudita de elaborada preparación y de improvisación. Como agrega Russ, la libertad del ensayo permite distinguir ambos géneros y ambos procedimientos, ya que una disertación filosófica designa una "demostraciónargumentación" rigurosa, un razonamiento controlado que desconoce la improvisación. El hilo lógico y demostrativo de la disertación filosófica no es en absoluto comparable con la ilación, mucho más libre, del ensayo. Si la progresión metódica y rigurosa diferencia el ensayo de la disertación filosófica, no obstante ambas tienen como objetivo común plantear una cuestión y un problema y querer resolverlos o, al menos, esforzarse en trabajar para su solución. Sus caminos, sin embargo, difieren: el ensayo quiere crear un clima que embargue al lector; la demostración quiere obtener su adhesión mediante un razonamiento riguroso, argumentado y progresivo;

(5) no es una demostración de tipo matemático. Esta negación implica, en primer lugar, definir el término demostración: designa una operación mental o un razonamiento deductivo destinado a establecer la verdad de una proposición a partir de premisas que se consideran verdaderas. Así, la demostración matemática parte de las premisas (axiomas, etc.) y se despliega obedeciendo las normas de la lógica. La demostración filosófica realmente no es asimilable a una demostración matemática, a una operación mental que establece deductivamente la verdad de una proposición, conectándola, mediante un vínculo necesario, con otras proposiciones evidentes. Esta distinción de géneros se impone por un doble motivo: por una parte, la disertación filosófica recurre a términos de lenguaje, ya sea corriente o especializado, cuyos significados no son unívocos. Por supuesto, estos términos designan conceptos y son fruto de un trabajo riguroso, pero la polisemia inherente al lenguaje puede ser fuente de ambigüedad. Por el contrario, los signos matemáticos están desprovistos de cualquier equivocidad. Por otra parte, la disertación filosófica descansa a la vez en una argumentación y en una demostración, en oposición al itinerario matemático, fundamentalmente demostrativo. Para Russ, en filosofía, no sólo se trata de demostrar, sino también de

14 El término "ensayo" en el sentido más modesto que se pueda definir, significa ensayar o ensayarse. Es decir, el ensayo podría ser definido como una manera de "actuar sin estar seguro del resultado" e inclusive "la acción de actuar en un campo por primera vez". De esta forma definido el ensayo corresponde a las implicaciones retóricas o expositivas de un intento arriesgado. 
argumentar, es decir, de poner en juego un conjunto de procedimientos que permitan hacer admitir una tesis. La especificidad del decurso filosófico se caracteriza por una autonomía que es ajena a la demostración matemática: en filosofía, la libertad de movimiento es manifiesta, mientras que en matemáticas, por el contrario, el desarrollo se efectúa a partir de datos mucho más coercitivos y se procede siguiendo un hilo lógico menos flexible que el de la argumentación filosófica;

(6) no es un comentario de texto. Recurriendo a Russ ${ }^{15}$, el ejercicio de comentario posee a la vez un parentesco profundo con la disertación y una especificidad que lo hace irreductible a esta última: aunque bajo cierta perspectiva, el método sea el mismo, no se trata en ningún caso de realizar una disertación sobre el autor. El comentario de texto se presenta en muchos aspectos como el espejo de la disertación. Se le propone al estudiante un fragmento filosófico y éste debe esclarecer el tema, la tesis o la idea directriz, la problemática constitutiva de dicho texto (se trata siempre de explicitar el problema filosófico suscitado y determinar su proceso de resolución) y los asuntos que están en juego. No obstante, a pesar de este parecido, según Russ, existe una diferencia real: en la disertación, los tribunales evaluadores exigen de los candidatos que realicen y lleven a cabo un trabajo muy personal (utilizando, evidentemente, los conocimientos filosóficos), mientras que el comentario de texto debe obedecer, sin ninguna paráfrasis, al pensamiento mismo del autor: no se trata, hablando con propiedad, de introducir ideas propias, sino de saber leer un texto y adaptarse a él con flexibilidad e inteligencia. Si la disertación es la escuela de la libertad del pensamiento, el comentario de texto designa la de la fidelidad al pensamiento, afirma Russ.

\section{La disertación como escrito}

Conviene entonces reiterar el sentido en que se puede entender la disertación. De lo que se habla aquí poco tiene que ver con la disertación puramente retórica del siglo XIX. No se trata tampoco de la disertación concebida como una especie de molde destinado a la elaboración de cualquier contenido, plan prefabricado como producto de una perversión de la retórica, reducida a una caricatura de sí misma.

Se trata entonces de una doble paradoja ${ }^{16}:<<$ hacer una disertación no es "disertar", y una disertación no es solamente un escrito, sino un diálogo. En efecto, una disertación es, en primer lugar, una respuesta fundamentada a una pregunta comprendida y no la ocasión de vastas elevaciones que se creen especulativas. Lo que implica una doble necesidad: la elucidación crítica de la pregunta planteada y una argumentación racional para responderla. En segundo lugar, la disertación es un diálogo. Se escribe alguna cosa para alguien; hay en ella un desafío y se trata de convencer al lector-corrector, de prevenir sus objeciones y de obtener su comprensión y, puede ser, su aceptación. Ella es también un escrito y un escrito filosófico que, como tal, une el pensamiento y la experiencia en la actividad mediadora del juicio. Comprender cual es la pregunta planteada, buscar darle una respuesta fundamentada, argumentada después de la

15 Ibid., p. 192-193.

16 Raffin, Françoise. La dissertation philosophique. La didactique á l'oeuvre. INRP-CNDP, Hachette, Paris, 1994, Pp. 89-90. (Traducción libre mía) 
reflexión y la deliberación es, como se aprecia, un ejercicio cuyo campo de aplicación no es solamente la prueba de filosofía en el examen de bachillerato, ni la sola filosofía. Un ejercicio de racionalidad presenta un interés que desborda ampliamente los campos escolar y universitario. >>

Entonces considerar la disertación en filosofía como un texto, como un discurso fijado por la escritura, nos lleva a reflexionar sobre el sentido y la importancia de este trabajo de escritura con relación a la exigencia filosófica. Es necesario determinar la especificidad de todo escrito filosófico entre los cuales figura la disertación. Reflexionaremos, en una palabra, sobre la disertación, no tanto como ejercicio sino como experiencia de estar en el mundo, de sí mismo y del pensamiento.

Como trabajo de escritura, la disertación implica un proceso de esfuerzo sobre sí mismo y, claro está, de distanciamiento con relación a sí mismo, por mínimo que sea ese distanciamiento. Sin duda, este trabajo de oponerse a sí mismo, sitúa al autor más allá de la inmediatez y de la ingenuidad elemental. Se trata de un desprenderse de la nostalgia del sí mismo (de ir más allá del estar contento de ser, y recibir sin más lo masivamente aceptado; superar ese sí mismo que a menudo se vuelve impermeable a los razonamientos y los argumentos), es decir, se pretende comprender lo que esta reivindicación de desprendimiento tiene de problemática. Es darle derecho al para sí, mediante el cual serán posibles la exigencia de verdad (y en consecuencia la superación de la simple certidumbre subjetiva) y la libertad.

\subsection{Lo escrito, vida del pensamiento}

Lo escrito es la no-muerte, es también la vida del pensamiento: es ese modo de exteriorización que lo hace posible.

Es en las palabras como nosotros pensamos, las palabras son de esta forma el elemento del pensamiento, su medio, no de expresión, sino de constitución, lo que hace que nosotros partamos de "pensamientos determinados y reales", que tengamos conciencia de lo que pensamos, y de que no se trata de un vago sentimiento pretendido más o menos inefable. No hay pensamiento que tome forma y cuerpo sin esta objetivación, esta exteriorización que permiten las palabras, las cuales son un modo de generalidad y abstracción, y del desbordamiento de la simple particularidad, de la simple inmediatez. Suponiendo la puesta en palabras, el pensamiento no puede entonces ser la pura presentación de una interioridad, de una intimidad (por más sentido que ella tenga). El problema de la verdad y de la falsedad del pensamiento no se plantea todavía, pero pensar, no es contentarse con sentir y confundirse consigo mismo, desaparecer en sí: es, al contrario, comenzar por desmarcarse de sí mismo. Por esta Entäusserung ${ }^{17}$ necesaria, pensar es exponer y exponerse, arriesgarse.

Escribir acentúa este esfuerzo, este efecto de desbordamiento de sí mismo y de lo que no es más que sentido, incluso si no se trata más que de expresar sin atribuir aún a la exigencia de verdad. Pero lo escrito no es por tanto la simple continuación de la

17 En alemán: Entäusserung. Enajenación, abandono; venta (Amador Hand Wörrterbuch. Deutsch-Spanisch und Spanisch-Deustch. Editorial Ramón Sopena. Barcelona, 1981. p.220) 
palabra, su simple fijación o trascripción. De hecho, lo escrito abre otras posibilidades; un nuevo dominio analítico de lo que es dicho (por esa linealidad de los símbolos), pero también del decir y del querer decir; así como la posibilidad de conservar el discurso, de hacerlo disponible a la memoria individual y colectiva, de volver a él, de retomarlo y de retomarse. Escribir supone entonces el reconocimiento de una ausencia y una insatisfacción.

En este sentido, sería pertinente aconsejar a los alumnos que cuando escriban tengan:

(a) un cuaderno personal donde consignen lo que les ha podido interesar, sorprender, escandalizar (palabras, encuentros, acontecimientos de actualidad o hechos naturales...); una existencia que, de otra manera, sería dispersa y sin línea de fuerza, sin línea de continuidad de los hechos;

(b) un cuaderno de diario filosófico en donde mencionen lo que juzgan ser las ideas fuerza que han encontrado en el curso, sin otra precisión histórica o de corpus, sino donde expliquen las razones de este interés; una manera para ellos de dejarse coger por las ideas y de disponerse para el pensamiento.

\subsection{La triple autonomía semántica del discurso}

De otra parte, como lo destaca Paul Ricoeur, gracias a la escritura el discurso adquiere una triple autonomía:

(a) Autonomía con relación al autor, sus intenciones, su presencia psicológica y carnal, sus efectos de retórica (mímica, entonaciones, gestos): "Lo que el texto significa no coincide más con lo que el autor ha querido decir. Significación textual y significación mental, es decir, psicológica, tienen sin embargo destinos diferentes." 18

(b) Autonomía con relación a aquel que recibe el texto: "el discurso escrito se suscita en un público que se extiende virtualmente a cualquiera que sepa leer" ${ }^{\prime 19}$

(c) Autonomía con relación al juego de circunstancias económicas, sociales, culturales de la producción del texto.

Lo escrito permite de esta manera alejarse de los límites del diálogo cara a cara; él permite apartarse de un cierto modo (reivindicación o nostalgia) de la inmediatez, de los juegos de la subjetividad y de la intersubjetividad de las circunstancias. Esta autonomía del texto, este distanciamiento no son "el producto de la metodología y no son, digamos, cosa sobreañadida...: ellos son "constitutivos del fenómeno del texto escrito." ${ }^{20}$ No es que el discurso no tenga relación alguna con las condiciones psicológicas y sociales; también, en lo que se refiere a las disertaciones, continuamos teniendo frente a nosotros, en clase, los alumnos que las hacen, que las escriben; pero no son en primer lugar las condiciones del acontecimiento del discurso escrito que queremos comprender aquí, sino su significación misma. Y precisamente, en lo escrito,

18 Ricoeur, Paul. Du texte á l'action. Essais d'herméneutique II. Seuil, Paris, p.111.

19 Ibíd., p. 111.

20 Ibíd., p. 112. 
“el discurso se supera, en tanto que acontecimiento, en la significación... Si la lengua es un meinen ${ }^{21}$, una pretensión significante, es precisamente en virtud de esta superación del acontecimiento en la significación" ${ }^{22}$

Este distanciamiento, constitutivo de lo escrito, no se identifica pura y simplemente, con la ruptura filosófica: él participa por tanto en su efectuar permitiendo desligarse de la particularidad de los individuos y de las circunstancias.

\section{La disertación como escrito filosófico}

\subsection{El problema del destinatario}

Esta autonomía estructural de lo escrito no impide por lo tanto que con relación a la redacción de la disertación en filosofía se plantee el problema del destinatario.

Hasta ahora hemos, con Paul Ricoeur, centrado nuestra atención sobre lo escrito en general y justo hasta esta consecuencia: "la liberación de la cosa escrita frente a la condición dialógica del discurso". Sin embargo, es necesario aquí la introducción de una diferencia: se debe distinguir entre el texto de ficción y el texto filosófico. Puesto que hay en este último un reconocimiento de derecho del interlocutor. Filosofar -y es esto mismo que se pide en una disertación- es tomar lugar en una comunidad hablante y pensante, escena humana que implica qué lugar se acuerda al otro y un "reparto de la voz" ${ }^{23}$. El interlocutor como principio de derecho, hace al otro parte de la instancia fundadora del discurso filosófico.

Filosofar es prever las objeciones, hacer siempre como si pudiera ser cuestionado por los otros.

Así hacía Descartes cuando en las Meditaciones metafísicas, en el Prefacio al lector, vuelve sobre las objeciones planteadas a propósito del Discurso del método; y advierte que responderá a las objeciones hechas por personas a las cuales remitió el texto; y donde llama a aquellos que quisieron con él: "meditar seriamente, y que podrían extraer su espíritu del comercio de los sentidos y desprenderse totalmente de toda clase de prejuicios" 24 Porque este interlocutor de derecho del discurso filosófico no es el individuo en su particularidad, sino el ser de la razón. También así hacía Platón cuando definía el pensamiento como "El razonamiento y el discurso son, sin duda, la misma cosa, pero ¿no le hemos puesto a uno de ellos, que consiste en un diálogo interior y silencioso del alma consigo misma, el nombre de razonamiento?" ${ }^{25}$ y plantea

21 En alemán: Meinen. Pensar; opinar; juzgar; querer decir; proponerse, ser de parecer; estimar; tener para sí, entender: das will ich meinen! ¡digo! - meinend. p.a. opinante. (Amador Hand Wörrterbuch. Deutsch-Spanisch und Spanisch-Deustch. Editorial Ramón Sopena. Barcelona. 1981. p.410).

22 Ibíd., p. 105.

23 Nancy, Jean-Lucy. Le parte des voix, Galilée, Paris, 1982.

24 Descartes, René. Meditaciones metafísicas, Editorial Espasa-Calpe. Madrid, 1999. Traducción de Manuel García Morente.

25 Platón. Sofista, 263e.P. 471 En: Platón. Diálogos V. Parménides, Teeteto, Sofista, Política, Editorial Gredos, Madrid, 1992. Traducciones, introducciones y notas de María Isabel Santa Cruz, Álvaro Vallejo Campos, Néstor Luis Cordero. 
entonces este interlocutor de derecho, incluso este contradictor de derecho que somos para nosotros mismos. Pensar, no es monologar y asentir a sí mismo, sino consentir en nosotros a la diferencia instituida por el Logos, a esta exterioridad de nosotros en nosotros mismos. Pensar es en primer lugar un esfuerzo contra sí mismo, incluyendo la oposición de la opinión, en tanto que esta procede de una lógica de la afirmación de sí mismo en su situación.

Entonces en su disertación, el alumno debe: (a) prever objeciones y críticas posibles; (b) tener claramente consciencia de que él será leído, es decir, que otro intentará comprenderlo; esto no implica una estructura jerárquica sino dialógica.

Se trata, entonces, de no vivir la disertación en el modo del encierro, como un ejercicio que no vale sino por él mismo, del cual el alumno se debe deshacer "escolarmente" por un llenado fetichista. Es necesario entonces comprender que la filosofía se dirige a otro o si no ella no lo es; (c) respetar intelectualmente el destinatario, dando prueba de pedagogía, sensibilizando al destinatario en la problemática tratada.

\subsection{Especificidad del escrito filosófico}

Todo escrito filosófico, entre ellos la disertación, debe ser un buen escrito específico. Lo es entonces por varios factores:

(a) El escrito filosófico está animado por una exigencia de argumentación y de justificación. Un texto filosófico quiere establecer alguna cosa: esto es una necesidad (lo que no es el caso de un texto literario) y es sobre esto que es juzgado. La filosofía no se define en primer lugar por un campo particular, por un objeto de pensamiento; los textos no son en primer lugar filosóficos por lo que dicen, sino por la manera como lo dicen: por un trabajo de la razón, de elaboración de sus exigencias de verdad, de sentido, de fundamento. Es filosófico un discurso que no se contenta con reflexionar sobre alguna cosa, sino que reflexiona también sobre lo que él hace. La filosofía es este discurso que siempre debe dar cuenta y dar razón de sí mismo.

Importa entonces al pensamiento en acto en una disertación, de retomarse, de criticarse, de reformarse, de rendir cuenta de lo que él hace; de ser entonces, más que un discurso sobre alguna cosa, un discurso sobre sí mismo.

(b) El escrito filosófico está animado por una exigencia de claridad conceptual.

Con la condición de comprender de que no se trata de un problema lexical, que no sería entonces más que un problema técnico y de definición; sino de un problema de pensamiento: descubrir que generan problemas las palabras que desde siempre parecen evidentes (verdad, ciencias, moral, progreso, felicidad.), y determinar racionalmente lo que genera problema. Hay, desde luego, algunas palabras técnicas, pero la filosofía no es una lengua en la cual bastaría dominar el vocabulario, sino una relación con la lengua; se trata de comprender mejor lo que hablar quiere decir, a la vez, en lo que concierne a los términos empleados y el hecho mismo de hablar. La filosofía y las exigencias de la escritura filosófica nos recuerdan nuestros deberes hacia el lenguaje.

Se debe entonces tener cuidado de no emplear de manera apresurada las definiciones 
como un saber que no nos permitirá pensar de antemano. Vale mejor para el alumno partir de lo que él pensaba o creía pensar, de su experiencia, antes que de las definiciones que, lo más frecuente, no son más que un saber exterior que le impide exteriorizar el problema planteado o implicado. Se trata entonces, no tanto de definir de antemano los objetos y partir de las definiciones, sino de un encadenamiento que nos conduce a ellas procediendo al análisis de las representaciones y de los discursos de los hombres.

(c) El escrito filosófico está animado por una exigencia de explicitación. El discurso filosófico actualiza los presupuestos, expectativas, consideraciones, causas, consecuencias de una idea, de una tesis. Lo que corresponde a una exigencia con relación a sí mismo: comprender tan claramente como sea posible por qué se piensa de lo que se piensa. Y con relación a cualquier otro, en tanto que él piensa, la explicitación le permite tener mucho ascendiente sobre lo que está pensado y planteando; permite también poder comprender e interiorizar este tema incluso si el otro no lo comparte; aquí es donde encontraremos este principio esencial del escrito filosófico: el reconocimiento de un interlocutor posible porque, precisamente, hay participación en la comunidad de los hombres que piensan.

Esto implica que estamos frente a un real en una situación de poca evidencia y que concierne la relación con la existencia, la experiencia, a sí mismo, al otro, al mundo, afrontamos la duda y los problemas de la verdad, del sentido, de la finalidad. Ver, y por tanto, no darse prisa en creer, tal es este otro principio de la reflexión filosófica que debe guiar la elaboración de la disertación.

\section{La disertación como experiencia}

Disertación como experiencia, no solamente en el sentido en el que la conciencia afronta lo que es el otro, el exterior, sino también en el sentido donde la consciencia hace la experiencia y la prueba de sí misma.

\subsection{El sujeto y la racionalidad}

La disertación como escrito filosófico no es solamente un ejercicio (analizar una cuestión, plantear un problema, responder desarrollando una reflexión ordenada) que se podría hacer, pero con el riesgo de quedar siempre en el exterior, en el desconocimiento, la ignorancia o el rechazo de lo que debe producir esta reflexión. Este presupuesto no solo es aplicable a la racionalidad; también puede percibirse en el ejercicio de la disertación. Es necesario que el alumno y el estudiante se comprometa a buscar de qué manera él está implicado, cuestionado por el problema y el desafío que implica la disertación. Se trata en el mismo movimiento (y esta paradoja tiene filosóficamente un sentido) de desligarse de sí mismo, de oponerse a sí mismo y de emplearse a fondo y arriesgarse. Este cuestionamiento de sí mismo supone la aceptación de una carencia primera, la cual muchas veces se esconde o protege con la ilusión de las técnicas de la disertación. La condición de la disertación como reflexión en acto se halla también, para el alumno, en el hecho de aventurarse en su elaboración. La disertación es de esta forma la huella de un esfuerzo sobre sí mismo. Aquí está implicada su libertad, y la disertación participa de esta liberación que prepara o 
madura para la libertad. Uno de los grandes obstáculos pedagógicos de la disertación es, en cierto sentido, el uso de los saberes (historia de la filosofía, reglas y consejos de método, variedad de referencias) para evitar pensar por sí mismo, esto es, entonces una manera más de abandonar su libertad. Sin embargo, es en esta experiencia donde nosotros volvemos a encontrar una de las mayores dificultades de la enseñanza filosófica: ¿cómo solicitar y demandar al estudiante libertad?

\subsection{La disertación como dialéctica y como fenomenología}

Haciendo prevalecer la exigencia de verdad, esto es, el trabajo de la razón, sobre la lógica de la afirmación de sí mismo y de sus intereses, la filosofía rompe con el mundo de la opinión y de la certidumbre subjetiva; ella rechaza esta inmediatez reivindicada que, en ocasiones en arte, reduce lo bello al consentimiento inmediato y que, en moral y en política, rechaza todo principio para desembocar en los relativismos y los escepticismos perezosos y confortables. Pero, quizás, todo no vale: ni todas las maneras de pensar y de vivir. ¿Y qué queda en un mundo falto de mediaciones? La moda: "En el sentido más amplio, (ella) es la única instancia reguladora autorizada por tal penuria ontológica. Tiene lugar de estética, de filosofía, de moral, de religión. Ella regla lo que se presenta como lo que se hace y se cree. Impone una necesidad en la contingencia y en lo arbitrario."26

Lamentablemente, es lo que constatamos con alguna frecuencia en nuestros alumnos, Pero, retomando la advertencia de Hegel, debemos cuidarnos de no caer de una ilusión a otra: en la ilusión del "buen sentido", en la ilusión de la proximidad, de la inmediatez (el sujeto permanece inmerso en lo que está por pensar), en la ilusión del raciocinio, de la especulación en el sentido negativo del termino, aquella de un sujeto separado y que ubicaría luego en un puro reino de ideas.

Así, contra un "platonismo vulgar", Dumont plantea que "lo que se supone de entrada de la filosofía, no es la separación, ruptura o corte con el mundo, sino, de una manera diferente, un entrar en razón, que permita tener una verdadera medida de los fenómenos y dar cuenta de ellos." ${ }^{27}$ Se trata, no de apartarse de la opinión, de los fenómenos, sino de dar razón de ellos. Y la disertación de filosofía, debe así dar lugar a este estar en el mundo del hombre, al arraigamiento, a nuestra condición y a sus tensiones, al cual nadie escapa, ni el profesor ni el alumno. Lo que implica es también darle un lugar al alumno y permitirle no ser externo a lo que debe ser pensado y a las razones por las cuales esto debe ser pensado. La disertación es entonces este camino que acoge las cuestiones de la verdad y del sentido, pero partiendo de los datos de nuestra condición, y no relegándolos en la irrealidad.

\subsection{El consentimiento a la mediación}

La disertación es consentimiento a la mediación y a este mundo del trabajo, de la paciencia y de la cultura por el cual se es humano.

26 Folscheid David. L'Esprit de l'athéisme et sa destine, Ed. Universitaires, Paris, 1991, p. 152 (Traducción libre mía).

27 Dumond Jean-Pierre. "Protreptique et initiation á la philosophie antique », Cahiers Eric Weil, n 2, Presses universitaires de Lille III, 1989, p.49. (Traducción libre mía). 
La mediación en filosofía no corresponde a la impaciencia y la nostalgia de la inmediatez, porque ella implica comprender, comprenderse, comprender al otro; la filosofía es despliegue, exposición, paciencia del concepto. La mediación da paso a lo humano, no como simple instrumento técnico, sino como fundamento de la humanidad del hombre.

¿Cuáles son entonces las exigencias de la mediación y qué debemos encontrar en una disertación?

La primera exigencia, para seguir a Folscheid ${ }^{28}$, es aquella de la diferencia. Pensar, es pensar las diferencias, estar atento a las diferencias sean ellas, aparentemente, las sutilezas o matices. Estas deben ser lo contrario de la tontería que no atrapa las cosas sino de manera masiva y unívoca. La segunda exigencia es la de la alteridad, sin la cual "la diferencia ensombrece en una negatividad informe". La tercera exigencia es la de totalidad -la más delicada de elaborar. Esta exigencia es traicionada "en cuanto una esfera particular reivindica el privilegio de satisfacer ella sola las exigencias de la mediación", lo que verificamos con todas las formas de reduccionismo.

¿Cuáles son las consecuencias de este trabajo de la mediación para el sujeto, es decir para el alumno y el profesor? "No hay comprensión de sí que no sea mediatizada por los signos, los símbolos y los textos" como lo escribe Ricoeur ${ }^{29}$, lo que disipa la ilusión de un conocimiento intuitivo de sí mismo, de una transparencia del sujeto para sí mismo imponiendo a la comprensión de sí mismo el rodeo por la cultura y la reflexión. Puede ser también, y esta sería la paradoja de la mediación (al alejar interpone), ¿se debe renunciar a la idea de una mediación total, que llegaría a un término y considerar la identidad como un proceso nunca acabado? Las cosas comenzarían, por el sujeto, sin que un fin sea asignable, sin este fin formal que es la libertad.

\section{Cierre}

Estamos lejos aquí de la seguridad de la subjetividad a la cual la filosofía y su enseñanza deben enfrentarse y que es uno de los grandes obstáculos del trabajo filosófico que se propone la disertación. Porque:

(a) la disertación en filosofía debe ser explicada técnicamente a los alumnos, pero ella debe también ser explicada filosóficamente, la disertación no tiene solo el sentido de un ejercicio escolar, sino también, el de una experiencia personal del pensamiento a partir de las condiciones de estar en el mundo;

(b) La técnica, las reglas de la disertación -como en general la pedagogía de la filosofíadeben entonces ser deducidas de la filosofía misma, es decir, del acto de filosofar.

(c) La disertación en filosofía es esencial a la enseñanza de la filosofía: al ofrecer un lugar a un destinatario de derecho, ella participa de la comunidad filosofante como "partición de las voces"; porque ella es esfuerzo de construcción racional, de argumentación, de análisis, todo esto supone no una respuesta ya existente que se

28 Op.cit. (Traducción libre mía).

29 Ricoeur P., «Ce qui me préoccupe», Esprit, avril/sept. 86, p. 239. (Traducción libre mía). 
debería volver a encontrar y conformarse con ella, sino un trabajo filosófico libre; por ser ella un esfuerzo para pensar, a partir de un tiempo y de un lugar sin ser prisioneros de estos: y porque ella ayuda a desbaratar los sueños de inmediatez y las trampas de la subjetividad.

Así como en alguna ocasión el profesor francés Jean-Jacques Guinchard ${ }^{30}$ preguntó algo respecto a uno sus escritos y exposiciones orales, quisiera entonces terminar valiéndome de sus palabras y preguntar: "¿lo que usted acaba de escuchar y leer es: (a) una disertación; (b) una disertación filosófica, o bien (c) alguna cosa que tiene el sello, bueno o malo, de la disertación?".

\section{Bibliografía}

Amador Hand Wörterbuch. Deutsch-Spanisch und Spanisch-Deustch. Editorial Ramón Sopena. Barcelona.

Descartes, René. Meditaciones metafísicas, Editorial Espasa-Calpe. Madrid, 1999. Traducción de Manuel García Morente.

Dumond Jean-Pierre. "Protreptique et initiation á la philosophie antique », Cahiers Eric Weil, $\mathrm{n}^{\circ} 2$, Presses universitaires de Lille III, 1989.

Folscheid David. L'Esprit de l'athéisme et sa destine, Ed. Universitaires, Paris.

Guinchard, Jean-Jacques. «Faut-il sauver la dissertation ?» en: L'agora. Revue Internationale de didactique de la philosophie. $\mathrm{N}^{\circ}$ 11. 2001.

Nancy, Jean-Lucy. Le parte des voix, Galilée, Paris, 1982.

Onfray, Michel. Antimanuel de philosophie. Editions Breal. Paris. 2005.

Platón. Diálogos V. Parménides, Teeteto, Sofista, Política, Editorial Gredos, Madrid, 1992. Traducciones, introducciones y notas de María Isabel Santa Cruz, Álvaro Vallejo Campos y Néstor Luis Cordero.

Raffin, F (Coord) La dissertation philosophique. La didactique á l'oeuvre. INRP, CNDP, Hachette, Paris. 1994.

Ricoeur P., «Ce qui me préoccupe», Esprit, avril/sept. 1986.

Ricoeur, Paul. Du texte á l'action. Essais d'herméneutique II. Seuil, Paris.

Russ, J., Los métodos en filosofía. Editorial Síntesis, Madrid, 2001, P. 93. Traducción y adaptación de Francisco Javier Vidarte.

Tozzi, Michel “Diversifier l'écriture philosophique" en: L'agora. Revue Internationale de didactique de la philosophie. $N^{\circ}$ 7. 2000.

Recibido: Junio 5 de 2009 - Aprobado: Agosto 15 de 2009

30 Guinchard, Jean-Jacques. «Faut-il sauver la dissertation ?» en: L'agora. Revue Internationale de didactique de la philosophie. $\mathrm{N}^{\circ}$ 11. 2001. (Recuperado el 9 de agosto de 2007: htttp://www.crdp montpellier.fr/ressources/agora/DO11024A.HTML) (Traducción libre mía). 